\title{
Video-Thoracoscopic Extended Thymectomy with Sternal Elevation and Carbon Dioxide Insufflation
}

\author{
Fukai $\mathrm{R}^{* 1}$, Inoue $\mathrm{T}^{2}$, Araki $\mathrm{O}^{3}$, Shiono $\mathrm{H}^{4}$ and Watanabe $\mathrm{K}^{1}$ \\ ${ }^{1}$ Department of General Thoracic Surgery, Shonan Kamakura General Hospital, Okamoto, Kamakura, Japan \\ ${ }^{2}$ Department of Cardiovascular and Thoracic Surgery, Dokkyo Medical University Koshigaya Hospital, Minami- \\ koshigaya, Koshigaya, Japan \\ ${ }^{3}$ Department of General Thoracic Surgery, Dokkyo Medical University, Kitakobayashi, Mibu, Japan \\ ${ }^{4}$ Department of General Thoracic Surgery, Nara Hospital Kinki University Faculty of Medicine, Otodacyo, Ikoma, \\ Japan
}

${ }^{*}$ Corresponding author: Fukai R, Department of General Thoracic Surgery, Shonan Kamakura General Hospital, 1370-1, Okamoto, Kamakura, Kanagawa, 247-8533, Japan, Fax: +81 46747 8243, Tel: +81 46746

1717, E-mail: r_fukai@shonankamakura.or.jp

Citation: Fukai R, Inoue T, Araki O, Shiono H, Watanabe K (2015) Video-Thoracoscopic Extended Thymectomy with Sternal Elevation and Carbon Dioxide Insufflation. J Case Rep Stud 3(4): 403. doi: 10.15744/2348-9820.3.403

Received Date: June 10, 2015 Accepted Date: August 08, 2015 Published Date: August 11, 2015

\begin{abstract}
To remove the thymus safely and completely in the surgical management for MG, especially for the upper pole of the thymus in the neck, we have performed video-thoracoscopic extended thymectomy (VATET) with sternal elevation and carbon dioxide $\left(\mathrm{CO}_{2}\right)$ insufflation. From November 2013 to May 2014, we conducted VATET for three MG patients. Symptomatic improvement was obtained, and there was neither mortality nor perioperative complications. VATET with sternal elevation and $\mathrm{CO}_{2}$ insufflation was a safe and valid technique for thymectomy for MG. Clinical short-term results were satisfactory.

Keywords: Sternal elevation; Carbon dioxide $\left(\mathrm{CO}_{2}\right)$ insufflation

Abbreviations: VATET: Video-Thoracoscopic Extended Thymectomy; MG: Myasthenia Gravis; VATS; Video-Assisted Thoracoscopic Surgery
\end{abstract}

\section{Introduction}

Thymectomy has been recognized as an effective surgical treatment for myasthenia gravis (MG). Recently, video-assisted thoracoscopic surgery (VATS) has been used for thymectomy in many institutions because of advances in minimally invasive surgery. It has been reported that thymic tissue can be found anywhere along the descending embryologic migration of the thymus, including in the neck [1]. Furthermore, a bilateral VATS approach for the resection of the upper pole of the thymus has been reported; this approach is therefore limited [2]. To ensure complete resection of thymic tissue, cervical thymic tissue as well as any mediastinal thymus should be removed in the surgical treatment for MG. In this paper, we present our video-thoracoscopic extended thymectomy (VATET) for MG with sternal elevation and carbon dioxide $\left(\mathrm{CO}_{2}\right)$ insufflation.

\section{Methods}

We performed our VATET procedure for three MG patients between November 2013 and May 2014 (Table 1). The female patients were 23 and 65 years old, and the male patient was 69 years old. The female patients both had symptoms, whereas the male patient with a thymoma showed only elevated acetylcholine receptor antibody level with no symptoms. According to the Myasthenia Gravis Foundation of America (MGFA) clinical classification, our female patients were classified as type IIa and type IIIb. They were treated previously in the neurology department of our institution, and the male patient received only a medical consultation.

\section{Operative indication}

Operative indication of VATET for MG patients with or without thymoma in our institution is as follows: (1) Diagnosed with MG by a neurologist and recommended for thymectomy by a neurologist; (2) MG with a positive anti-acetylcholine receptor antibody level and symptoms; (3) MG with a well-demarcated thymoma and a diameter of less than $5 \mathrm{~cm}$. 


\begin{tabular}{|c|c|c|c|}
\hline Age, Sex & 23, Female & 65 , Female & 68, Male \\
\hline MGFA $^{\star}$ Classification & IIa & IIa & None \\
\hline Symptoms & Tiredness, Ptosis & $\begin{array}{l}\text { Ptosis, Double vision, } \\
\text { Muscle weakness }\end{array}$ & None \\
\hline $\begin{array}{l}\text { Anti AchR Level Before/ } \\
\text { After operation (nmol/l) }\end{array}$ & $440 / 160$ & $55 / 3$ & $25 / 24$ \\
\hline Tumor Size & - & $4.0 \mathrm{X} 2.4 \mathrm{~cm}$ & $3.3 \mathrm{X} 2.1 \mathrm{~cm}$ \\
\hline Pathology & Thymic Hyperplasia & Thymoma AB & Thymoma A \\
\hline Masaoka Classification & None & II & I \\
\hline
\end{tabular}

${ }^{*}$ MGFA: Myasthenia Gravis Foundation of America Table 1: Patient characteristics

\section{Surgical technique}

After inducing general anesthesia, each patient was intubated with a double-lumen endotracheal tube with selective one-lung ventilation. They were placed in a supine position and elevated with surgical rolls to adequately expose the bilateral axillae. Informed consent was obtained from each patient.

The operation was started from the right side. A sealed $12 \mathrm{~mm}$ trocar through the fourth intercostal space (ICS) in the midaxillary line was inserted, and then a $10 \mathrm{~mm}$ thoracoscope was placed. Carbon dioxide $\left(\mathrm{CO}_{2}\right)$ was insufflated at $8 \mathrm{~cm} \mathrm{H}_{2} \mathrm{O}$, which was the minimum pressure to obtain sufficient visualization of the mediastinal surgical field immediately after the beginning of the operation, because the right lung is rapidly collapsed by the effect of carbon dioxide insufflation. After inspection of the right thoracic cavity, two additional $5 \mathrm{~mm}$ trocars were inserted as operative ports: one through the third ICS in the anterior axillary line and one through the fifth ICS in the anterior axillary line (Figure 1).
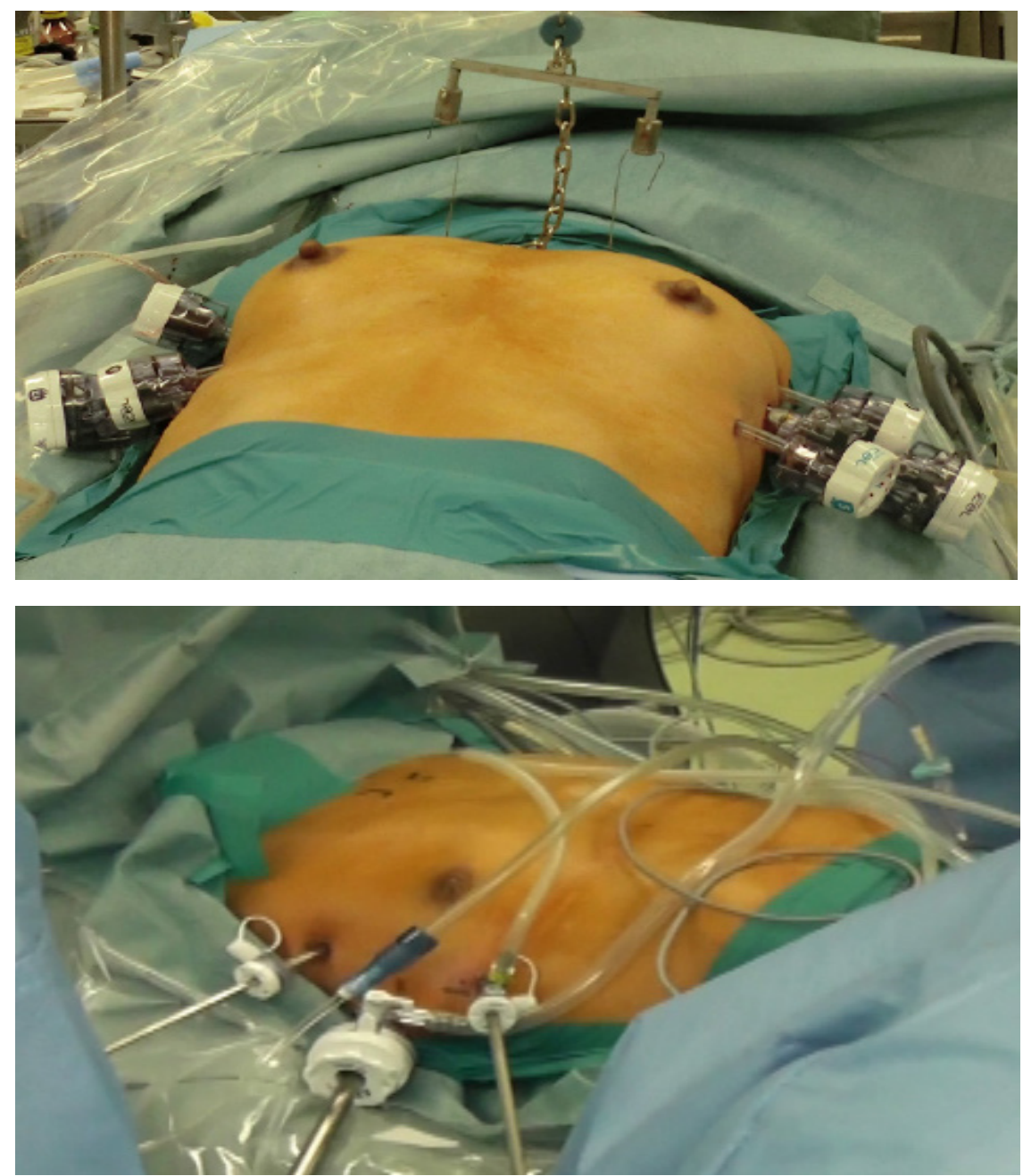

Figure 1: Port positions and lifting of the sternum during VATET. VATET, video-thoracoscopic extended thymectomy 
We exfoliated thymic tissue from the SVC, left innominate vein, pericardium, and the back of the sternum, and then reached the left mediastinal pleura, and branches of the thymic vein were divided with ultrasonic scissors. The left mediastinal pleura was not opened at this point to prevent injury of the left phrenic nerve. The right internal mammary vein was transected using ultrasonic scissors after ligation of the proximal side.

When dissection of the right mediastinal thymic lobe was almost finished, we inserted a Ring Wire ${ }^{\mathrm{TM}}$ (Micro Inc., Tokyo, Japan) through the first ICS for lifting the cephalad part of the sternum to open up the cervical area using a Lapa-Her-Closure ${ }^{\mathrm{TM}}$ device $^{-}$ (Hakko Inc., Tokyo, Japan) (Figure 2). With sufficient visualization of the lower neck by lifting the sternum, dissection of the cervical thymic tissue was performed from the left side.

\section{Sternal elevation}

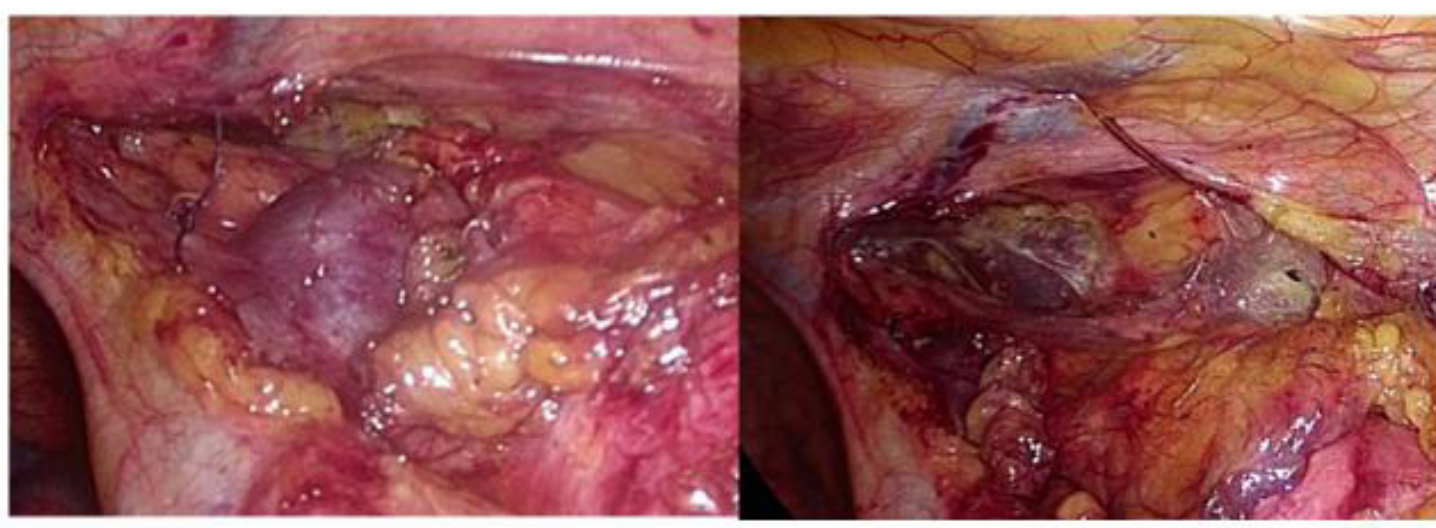

\section{Before}

After

Figure 2: After sternal elevation, the retrosternal space was enlarged

After the table was rotated from left to right, we inserted three ports on the left side similar to those on the right side. If the heart is obstructive using the left arm, we shifted the trocar in the fifth ICS from the anterior axillary line to the midclavicular line. The left mediastinal pleura was opened just above the left phrenic nerve and just below the sternum. At this point, the operative field from the left side was connected with that from the right side. The left innominate vein was identified, and the left cervical and mediastinal thymus with overlying left pericardiophrenic fat was dissected. Finally, dissection of the right cervical thymic tissue was performed, thus the entire cervical dissection clearly exposed the SVC, the right innominate artery, and the trachea (Figure 3 ). The resected thymic tissue was removed with an endobag. In the case of a thymoma, only one incision was extended to $4 \mathrm{~cm}$ to remove the specimen without capsular breakage.

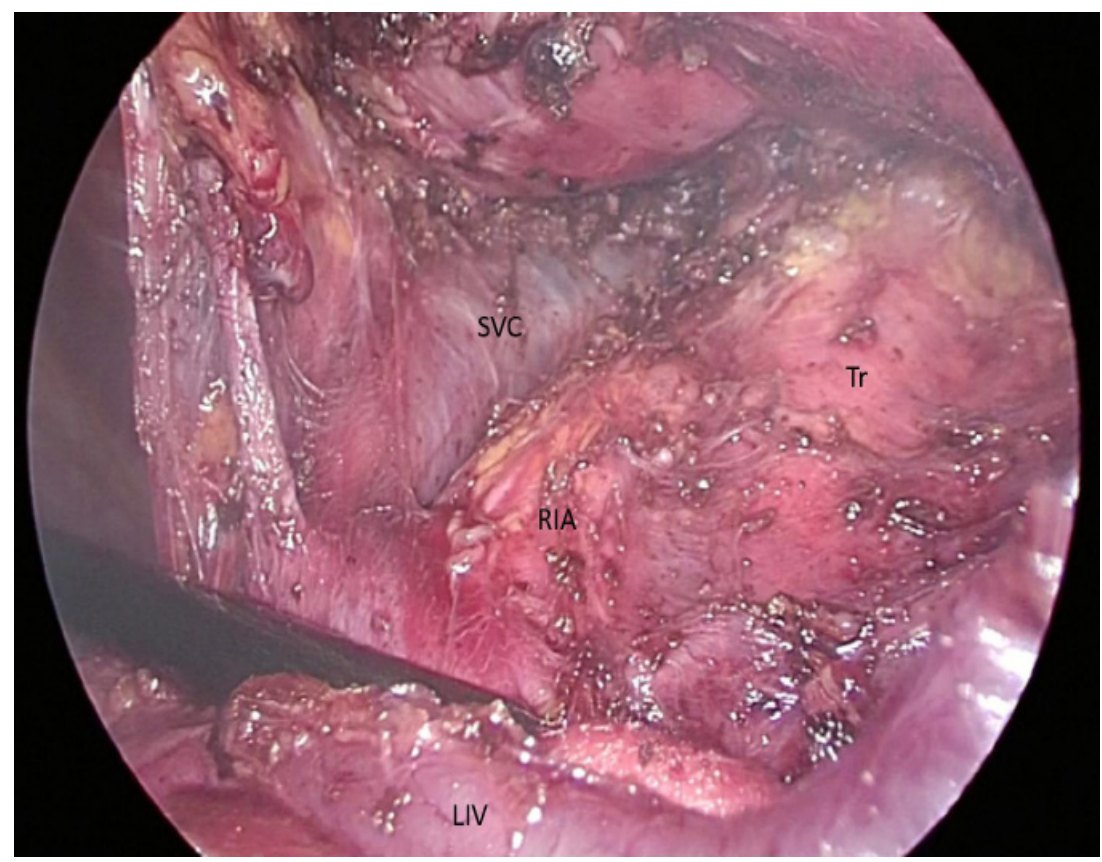

Figure 3: Surgical view after removal of cervical thymus and fat tissue. SVC: Superior Vena Cava; RIA: The Right Innominate Artery; LIV: The Left Innominate Vein; and Tr: Trachea 


\section{Results and Discussions}

The mean operative time was 185 minutes (range, 174 to 201 minutes), and blood loss was $20 \mathrm{ml}$ (range, 10 to $30 \mathrm{ml}$ ). The removed thymus specimens weighed 32-45 g. All patients were discharged or transferred to the department of neurology within one week after the operation, and their postoperative courses were uneventful. The World Health Organization histological classification of thymomas were type $\mathrm{A}$ (male, $50 \mathrm{~mm}$ ) and type $\mathrm{AB}$ (female, $31 \mathrm{~mm}$ ). The symptoms of the two female patients improved immediately after the operation, and their acetylcholine receptor antibody levels also decreased, from $480 \mathrm{to} 170 \mathrm{nmol} / \mathrm{ml}$ in the younger patient, and from 55 to $1.7 \mathrm{nmol} / \mathrm{ml}$ in the older patient.

Thymectomy is recognized as an effective treatment of autoimmune MG. VATS has recently been applied to more MG cases because of advances in minimally invasive surgery. On the other hand, it has been reported that thymic tissue can be found in the perithymic fat outside the boundary of the anatomic thymic lobes, as well as elsewhere in the mediastinum and the neck [1]. In fact, Shigemura et al reported that residual thymus had been found in the neck after bilateral VATS thymectomy without a transcervical approach [2]. Therefore, thoracic surgeons should aim to remove the entire thymus including any found in the neck. We have rounded out the technique of combined sternal elevation with a wire and carbon dioxide insufflation for a wider surgical field to include the neck, and our short-term results have been satisfactory.

There are two important points concerning VATET, the first being a wide surgical field to include the lower neck, the second being positioning of the patient. The thymus is distributed in a narrow flat space behind the sternum. Therefore, if a thoracoscopic approach is chosen, the surgeon encounters a tangential view of the left brachiocephalic vein and right innominate artery leading to an increased risk of bleeding. Consequently it is difficult to dissect the thymus with these structures in the field, particularly in the neck. It is reported that the positive pressure from carbon dioxide insufflation serves to open up the cervical area allowing dissection of the superior poles of the thymus [3]. We tried to ensure better surgical field of the upper thymic poles by lifting the sternum at the first intercostal level, for preventing the bleedings from above-mentioned vessels, thus it made the procedure safer and more confident.

Another important aspect of the surgery is patient positioning. In this operation, we place operative ports between the third to fifth intercostal spaces. Accordingly, if the arm of the patient is raised up, since it can interfere with the instrument which is employed through the third intercostal port, this port cannot be adequately used. Therefore, by elevating the chest of the patient with the surgical rolls, the surgeon has more working room. Thus, we can perform a bilateral VATS approach by rotating the operating table without changing the patient's position.

Surgical options for thymectomy include a trans-sternal approach, a transcervical approach, a combined transcervical-transsternal maximum thymectomy, and VATS [3-5]. Takeo et al. reported Video-assisted extended thymectomy in patients with thymoma by lifting the sternum [4]. They performed collar-shaped cervical incision $(4 \mathrm{~cm})$ and a transverse abdominal incision $(6-8 \mathrm{~cm})$ in addition to thoracoscopic approach, whereas we used only thoracoscopic incisions. However, their method was applied even to invasive thymoma cases, while we restricted to only non-invasive and less than $5 \mathrm{~cm}$ lesions. Recently, it has been reported that comparative clinical outcomes between extended trans-sternal and VATS are equivalent [5,6]. Short-term results of our patients are encouraging. Further investigation with long-term follow-up will be needed regarding the efficacy of this procedure.

\section{Conclusion}

VATET with sternal elevation and $\mathrm{CO}_{2}$ insufflation is a safe technique for thymectomy for MG with non-invasive thymoma. Further investigation with long-term follow-up will be needed regarding the efficacy of this procedure.

\section{Acknowledgment}

The authors thank Mizuki Tanaka, the medical clerk of Dokkyo Medical University Koshigaya Hospital, for industrious collecting medical information of the patients.

\section{References}

1. Masaoka A, Nagaoka Y, Kotake Y (1975) Distribution of thymic tissue at the anterior mediastinum. Current procedures in thymectomy. J Thorac Cardiovasc Surg 70: 747-54.

2. Shigemura N, Shiono H, Inoue M, Minami M, Ohta M, et al. (2006) Inclusion of the transcervical approach in video-assisted thoracoscopic extended thymectomy (VATET) for myasthenia gravis: a prospective trial. Surg Endosc 20: 1614-8.

3. Jaretzki A 3rd, Wolff M (1988) "Maximal” thymectomy for myasthenia gravis. Surgical anatomy and operative technique. J Thorac Cardiovasc Surg 96: 711-6.

4. Takeo S, Sakada T, Yano T (2001) Video-assisted extended thymectomy in patients with thymoma by lifting the sternum. Ann Thorac Surg 71: 1721-3.

5. Meyer DM, Herbert MA, Sobhani NC, Tavakolian P, Duncan A, et al. (2009) Comparative clinical outcomes of thymectomy for myasthenia gravis performed by extended transsternal and minimally invasive approaches. Ann Thorac Surg 87: 385-91.

6. Shiono H, Kadota Y, Hayashi A, Okumura M (2009) Comparison of outcomes after extended thymectomy for myasthenia gravis: bilateral thoracoscopic approach versus sternotomy. Surg Laparosc Endosc Percutan Tech 19: 424-7. 


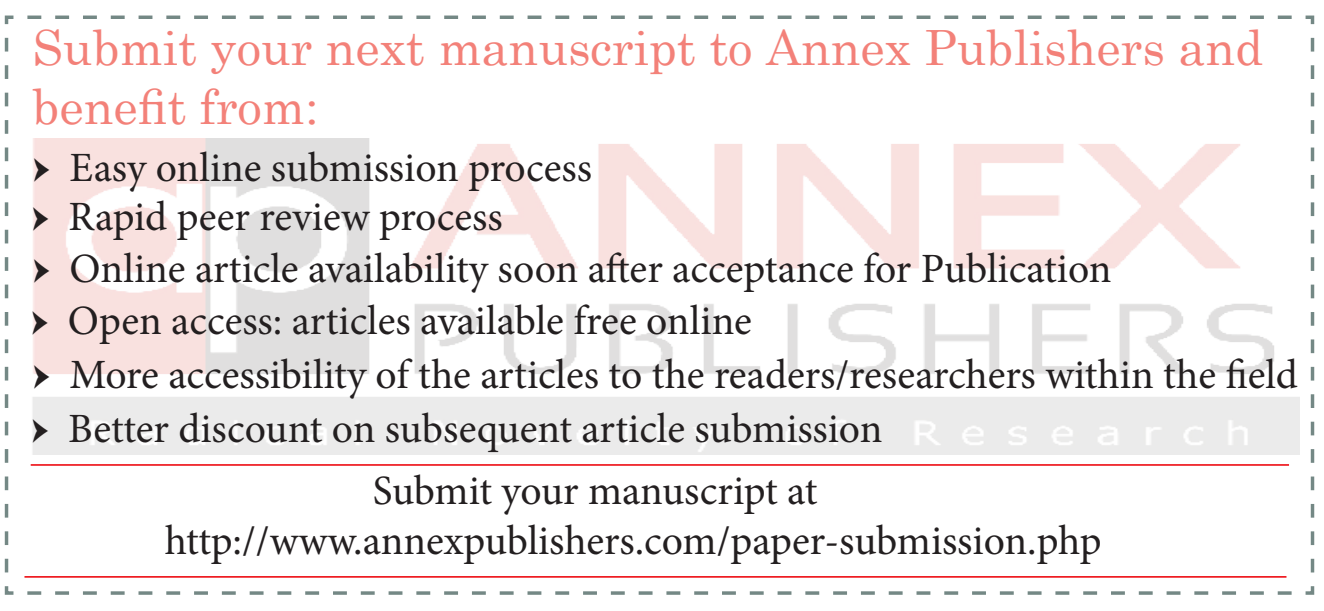

